\title{
Circadian Rhythms in Voiding Function and Dysfunction
}

\author{
Sehyung Cho, Dong-Hee Han, Dong-Hee Kim ${ }^{1}$ \\ Department of Physiology, Neurodegeneration Control Research Center, Kyung Hee University School of Medicine, Seoul; \\ ${ }^{1}$ Department of Ophthalmology, Konkuk University Chungju Hospital, Konkuk University School of Medicine, Chungju, Korea
}

\begin{abstract}
While circadian rhythms in fluid intake, urine production, and urine storage have been substantiated in diurnal human and nocturnal rodents, the mechanism(s) underlying it is largely unknown. With the elucidations of molecular clockwork and its functional significance in mammals, new opportunities arise to investigate possible circadian control of voiding function and dysfunction, which undoubtedly needs immediate attentions of researchers in the field.
\end{abstract}

Keywords: Voiding; Circadian; Clockwork; Sleep; Diurnal

Life on this rotating planet is confronted with periodic changes of its environment. Often, these changes are quite predictable like daily variations in environmental illumination, temperature, humidity, and food/predator availability. From an evolutionary perspective, organisms that can predict environmental changes and actively prepare themselves for these changes would have selective advantages over those who cannot. Indeed, endogenous mechanisms called circadian clocks have successfully emerged so that virtually all living things on Earth display circadian rhythms in their biochemistry, physiology, pathology, and behavior [1,2].

Consequently, most physiological functions are tightly locked to their activity/rest phases to ensure optimal performance. Day-active animals including humans consume most of their food during the day, while night-active animals like laboratory rodents during the night. Accordingly, water intake, urine production, and urine storage also display distinct circadian variations in diurnal human and nocturnal rodents. Intuitively, urine production and voiding must predominate during the active phase, whilst increased storage of urine in the bladder and reduced frequency in urination have to be ensured to promote better rest and sleep, which has been substantiated by research observations [3,4]. Moreover, failures of this homeostatic regulation are predicted to negatively affect organisms' well-being. Indeed, nocturia (bothersome waking-up at night to void) are known to decrease quality of life and negatively affect morbidity and mortality in human beings, especially in the elderly $[5,6]$. Researches also indicated that nocturia disrupts sleep architecture and predicts obstructive sleep apnea while acute sleep deprivation results in excess diuresis and natriuresis $[7,8]$.

Despite the ample evidence supporting clear circadian/diurnal variations in urine production and storage, the mechanism(s) underlying it is largely unknown. Now that molecular components of circadian clock have been identified and several mutant animal lines lacking in clock functions are available [1], novel approaches are needed to delineate whether voiding function and dysfunction are under the direct control of mammalian time-keeping system. This can be addressed by comparing clock-intact animals with those deficient either systemically or tissue-specifically in clock functions. In these efforts, key molecules linking circadian clock and voiding function should be clarified. Given the multifaceted nature of voiding function and dysfunction [5,9], however, all the possible alternatives need to be considered as well.

Since neural and humoral outputs of the suprachiasmatic nucleus (SCN), the master clock in mammals [10], orchestrate
Corresponding author: Sehyung Cho

Department of Physiology, Kyung Hee University School of Medicine,

Hoegi-dong, Dongdaemun-gu, Seoul 130-701, Korea

Tel: +82-2-961-0984 / Fax: +82-2-969-6343 / E-mail: sehyung@khu.ac.kr

Submitted: March 1, 2011 / Accepted after revision: March 20, 2011
This is an Open Access article distributed under the terms of the Creative Commons Attribution Non-Commercial License (http://creativecommons.org/licenses/by-nc/3.0/) which permits unrestricted non-commercial use, distribution, and reproduction in any medium, provided the original work is properly cited. 
peripheral clocks present throughout the body, micturition centers in the brain [11] and local urinary functions in the kidney and bladder can be direct targets of circadian oscillatordriven hormones and neural activities. In addition, possible local clocks residing in the kidney and bladder may derive time of day-dependent urinary functions.

One crucial thing that should not be forgotten in designing experiments and interpreting their results is that we humans are diurnal while experimental model animals used in most urological researches are nocturnal. While a few similarities (i.e., increased neural activities of the $\mathrm{SCN}$ during the daytime and melatonin secretion during the night) are observed, lots of neuroendocrine and behavioral functions are completely reversed in diurnal and nocturnal mammals [12].

Considering that urological problems are one of the major complaints of the elderly in this aged society, and that lots of workers in the developed and developing countries are subjected to various shift work scheduling and circadian disruption, possible circadian controls of voiding function and dysfunction is undoubtedly an urgent agenda that requires immediate attentions of the researchers in the field.

\section{REFERENCES}

1. Takahashi JS, Hong HK, Ko CH, McDearmon EL. The genetics of mammalian circadian order and disorder: implications for physiology and disease. Nat Rev Genet 2008;9:764-75.

2. Dibner C, Schibler U, Albrecht U. The mammalian circadian timing system: organization and coordination of central and peripheral clocks. Annu Rev Physiol 2010;72:517-49.
3. Van Hoeck K, Bael A, Lax H, Hirche H, van Gool JD. Circadian variation of voided volume in normal school-age children. Eur J Pediatr 2007;166:579-84.

4. Herrera GM, Meredith AL. Diurnal variation in urodynamics of rat. PLoS One 2010;5:e12298.

5. Nørgaard JP, Hashim H, Malmberg L, Robinson D. Antidiuresis therapy: mechanism of action and clinical implications. Neurourol Urodyn 2007;26:1008-13.

6. Kujubu DA, Aboseif SR. An overview of nocturia and the syndrome of nocturnal polyuria in the elderly. Nat Clin Pract Nephrol 2008;4: 426-35.

7. Kaynak H, Kaynak D, Oztura I. Does frequency of nocturnal urination reflect the severity of sleep-disordered breathing? J Sleep Res 2004;13:173-6.

8. Kamperis K, Hagstroem S, Radvanska E, Rittig S, Djurhuus JC. Excess diuresis and natriuresis during acute sleep deprivation in healthy adults. Am J Physiol Renal Physiol 2010;299:F404-11.

9. Chang SC, Lin AT, Chen KK, Chang LS. Multifactorial nature of male nocturia. Urology 2006;67:541-4.

10. van Esseveldt KE, Lehman MN, Boer GJ. The suprachiasmatic nucleus and the circadian time-keeping system revisited. Brain Res Brain Res Rev 2000;33:34-77.

11. Kim SE, Ko IG, Kim BK, Sung YH, Shin MS, Cho S, et al. Transplantation of human adipose-derived stem cells into the urethra ameliorates stress urinary incontinence and blunts the induction of c-Fos immunoreactivities in brain areas related to micturition in female rats. Anim Cells Syst 2010;14:237-44.

12. Challet E. Minireview: entrainment of the suprachiasmatic clockwork in diurnal and nocturnal mammals. Endocrinology 2007;148: 5648-55. 